\title{
A Review of Cytokine Storm in COVID-19
}

\author{
Meenakshi $^{1}$, Amit Samadhiya $^{2}$, Monil Singhai ${ }^{3}$, Rajeev Goyal $^{4}$
}

\begin{abstract}
Aim: To review pathogenesis of cytokine storm in COVID-19 cases.

Background: Human coronaviruses ( $\mathrm{hCoV}$ ) mainly infect upper airways and cause seasonal mild to moderate cold-like respiratory symptoms or severe pneumonia leading to fatal acute lung injury (ALI) and acute respiratory distress syndrome (ARDS). Recent clinical investigation reveals that mild COVID-19 patients had high level of IL1B, IFN- $\gamma$, CXCL10/IP-10, and CCL2/MCP-1, while patients requiring ICU admission had higher level of GCSF, CXCL10/IP-10, CCL2/MCP-1, and CCL3/MIP-1A, indicating inflammatory cytokine release is critical in COVID-19 progression. IL-1 and IL- 6 are responsible for elevation of acute-phase reactants, such as C-reactive protein, serum amyloid A, fibrinogen, hepcidin, and inhibition of albumin synthesis which all together causes a procoagulant environment. In a meta-analysis including nine studies (total 1426 patients), mean IL-6 levels were more than 3 times higher in patients with complicated COVID-19 compared to those with uncomplicated disease, and high IL-6 levels were associated with mortality risk. IL-6 is an important marker of inflammation and can guide the clinicians in recognizing patients with severe COVID-19 early in the disease course. Delay in the interferon release and presence of LPS by secondary bacterial infections increases the severity of cytokine storm. Thus, commencement of early broad-spectrum antibiotic course is advisable. Cytokine storm appears after first week of symptoms when viral load starts decreasing, and this indicates the immunopathogenesis of the ARDS.

Conclusion: The aberrant release of multiple cytokines in COVID-19 produces immunopathogenic damage to tissues and organs, even while the immune response tries to overcome the evading mechanisms of virus. Delay in the interferon release and presence of lipopolysaccharide by secondary bacterial infections increase the severity of cytokine storm. IL-6 could be used as a potential marker for severity of the ARDS. However, anti-IL6 antibody Tocilizumab failed to prove effective in clinical trials. Corticosteroid therapy is being given for moderate and severe cases of ARDS, but it needs a very fine balance to outweigh immunosuppressive effects.

Keywords: COVID-19, Cytokine storm, Inflammation.

Indian Journal of Medical Biochemistry (2020): 10.5005/jp-journals-10054-0151
\end{abstract}

\section{INTRODUCTION}

Severe acute respiratory syndrome coronavirus 2 (SARS-CoV-2), which causes COVID 19, became a major threat to public health and has now emerged as a pandemic. Coronaviruses belong to the Coronaviridae family which are enveloped, positive-strand RNA viruses. Human coronaviruses (hCoV) mainly infect upper airways and cause seasonal mild to moderate cold-like respiratory symptoms or severe pneumonia leading to fatal acute lung injury (ALI) and acute respiratory distress syndrome (ARDS). ${ }^{1,2}$ Severity of the disease is influenced by interplay between several factors such as host immune response, virus natural history and transmission (viral shedding in different stages of clinical illness), age and comorbid conditions such as diabetes, obesity, heart failure, and renal failure of the infected person, etc. Highly pathogenic hCoVs have been responsible for various epidemics in the past and pose a substantial threat to public health.

In context of an outbreak or pandemic, it is extremely important to know the clinical course of the disease along with the common and uncommon presentations. A rapid and well-coordinated innate immune response is the first line of defense against viral infections, but dysregulated excessive immune responses may cause immunopathology. Cytokines and chemokines have important roles in immunity and immunopathology during viral infections.

\section{Tropism From the Lung to Multiple Organs}

SARS-CoV-2 makes its entry through a zinc-containing metalloenzyme ACE2, which is reportedly present on type I and type II pneumocytes, enterocytes of all parts of the small intestine,
1,2,4 Department of Biochemistry, Lady Hardinge Medical College, New Delhi, India

${ }^{3}$ Center for Arboviral and Zoonotic Diseases, National Center for Disease Control, Delhi, India

Corresponding Author: Rajeev Goyal, Department of Biochemistry, Lady Hardinge Medical College, New Delhi, India, Phone: +91 8191861234, e-mail:docrajeev@gmail.com

How to cite this article: Meenakshi, Samadhiya A, Singhai $M$, et al. A Review of Cytokine Storm in COVID-19. Indian J Med Biochem 2020;24(2):59-61.

Source of support: Nil

Conflict of interest: None

the brush border of the proximal tubular cells of the kidney, and the endothelial cells of small and large arteries and arterial smooth muscle cells, suggesting that in SARS, there is widespread extrapulmonary dissemination of virus, resulting in viral shedding in respiratory secretions, stools, urine, and possibly even in sweat. ${ }^{3}$

It has been postulated that a preceding infection by another pathogen may facilitate SARS-CoV infection by damaging the upper respiratory tract before spreading to involve the lung. This may partly explain the longer incubation period of SARS-CoV when compared to influenza. ${ }^{4}$

\section{Cytokine Storm}

The inflammatory response in viral pneumonia can be a doubleedged sword. Although beneficial inflammation is necessary for the local tissues to fight infection, exacerbated inflammatory

(-) The Author(s). 2020 Open Access This article is distributed under the terms of the Creative Commons Attribution 4.0 International License (https://creativecommons. org/licenses/by-nc/4.0/), which permits unrestricted use, distribution, and non-commercial reproduction in any medium, provided you give appropriate credit to the original author(s) and the source, provide a link to the Creative Commons license, and indicate if changes were made. The Creative Commons Public Domain Dedication waiver (http://creativecommons.org/publicdomain/zero/1.0/) applies to the data made available in this article, unless otherwise stated. 
responses in pneumonia patients result in excessive release of proinflammatory cytokines known as "cytokine storm", leading to detrimental outcomes such as diffuse alveolar damage and fibrosis, progressive respiratory failure, and multiple organ dysfunction. ${ }^{5-7}$ Cytokine storm is a severe immune condition characterized by rapid proliferation and hyperactivation of T cells, macrophages, natural killer cells, and overproduction of more than 150 inflammatory cytokines and chemical mediators by immune or non-immune cells. ${ }^{5,8,9}$

Cytokine storms can be caused by a number of infectious and non-infectious etiologies, especially viral respiratory infections such as H5N1 influenza, SARS-CoV-1, and SARS-CoV-2. Other causative agents include the Epstein-Barr virus, cytomegalovirus, and group A streptococcus and non-infectious conditions such as graft-vs-host disease. We further study the fundamental aspects of cytokine release after SARS-CoV-2 infection and its interaction with host innate immunity.

\section{SARS-CoV-2 and Innate Immune System}

Viral RNAs are recognized by the innate immune system through three major classes of cytoplasmic pattern recognition receptors (PRRs): Toll-like receptors (TLRs), RIG-I-like receptors(RLRs), and NOD-like receptors (NLRs), which trigger the expression of interferon (IFN) and activation of antiviral effectors such as Natural Killer cells, T CD8+ cells, and macrophages. ${ }^{10}$

TLR7 and TLR9, which are located in the endosomes, recognize ssRNA of SARS-CoV 2 and signal the activation of the transcription factor IRF7, which increases the expression of both type I and type II interferons. Similar to interferons, transcription factor NF-kB, which regulates genes responsible for both the innate and adaptive immune response, is upregulated by TLR-activated pathways. It is an important transcription factor for the production of pro-inflammatory cytokines, including interleukin-1 (IL-1), IL-6, and tumor necrosis factor-alpha (TNF-a), and of chemokines, such as IL-8, C-C motif chemokine ligand 2 (CCL2), and C-X-C motif chemokine 10 (CXCL10). ${ }^{11}$

Coronaviruses, like all other successful viruses, have developed strategies to counter the innate immune response. Expression of IFN is a crucial component of initial immune response. The nucleocapsid protein ( $\mathrm{N}$ protein) which is important for the encapsidation of SARS-CoV 2 viral RNA, acts as an interferon (IFN) antagonist.

Viruses appear to be invisible to intracellular viral sensors (such as RIG-I, MDA5, and TLR3, 7, 9) because double-stranded RNA, which is synthesized during viral replication and is a potent stimulator of the innate immune system, is buried in double-membrane vesicles (DMV). ${ }^{12}$ Additionally, viral proteins, in particular nsp1, nsp3, N protein, and the SARS-CoV accessory proteins ORF6 and ORF3b, also prevent IFN induction. ${ }^{13-17}$ SARS-CoV and MHV (Murine Hepatitis Virus) nsp1 function, at least in part, by degrading host cell mRNA and inhibiting translation. ${ }^{18}$ Overexpression of $7 a$, a protein specifically encoded by SARS-CoV, has been shown to induce apoptosis via a caspase-dependent pathway in cell lines derived from different organs, including lung, liver, and kidney. ${ }^{19}$ Increase in the apoptosis explains the lymphopenia seen in COVID-19 patients.

\section{Cytokine Storm Caused by SARS-CoV-2}

At the early stages of SARS-CoV infection, release of cytokines and chemokines occurs from respiratory epithelial cells, dendritic cells (DCs), and macrophages. These cytokines are responsible for the development of clinical symptoms such as fever, chills, headaches, dizziness, and fatigue. TNF-a can cause flu-like symptoms like fever, general malaise, and fatigue along with vascular leakage, cardiomyopathy, lung injury and acute-phase protein synthesis. ${ }^{5}$

Recent clinical investigation reveals that mild COVID-19 patients had high level of IL1B, IFN $\gamma$, CXCL10/IP-10, and CCL2/ MCP-1, while patients requiring ICU admission had higher level of GCSF, CXCL10/IP-10, CCL2/MCP-1, and CCL3/MIP-1A, indicating inflammatory cytokine release is critical in COVID-19 progression. ${ }^{5}$ Channappanavar et al. showed that robust virus replication and delayed IFN-I signaling promote severe disease. IFN-I promotes the accumulation of monocyte macrophages, resulting in lung damage, vascular leakage, and suboptimal T cell responses. ${ }^{20}$

However, cytokines and chemokines measurement in the plasma from SARS patients are difficult to interpret partly because of the many confounding factors during the disease course. Administration of the anti-IL-6-Ra antibody tocilizumab has been proposed as a treatment for COVID-19 patients. However, very recently, Hoffmann-La Roche has announced disappointing results from its much-anticipated phase 3 COVACTA trial of tocilizumab, raising questions about the efficacy of interleukin (IL)- 6 blockade in patients with severe COVID-19 pneumonia. ${ }^{21}$

IL-1 is a key cytokine that is responsible for proinflammatory activity. Along with IL-1, TNF and chemotactic cytokines IL-8 and MCP-1 also appear in the early hours after infection and stimulate the production of IL-6. IL-8 can be secreted by any cells with toll-like receptors that are involved in the innate immune response. Usually, it is the macrophages that encounter an antigen first and thus are the first cells to release IL-8 to recruit other cells.

Steroids are also known to inhibit the gene expression of IL-6, IFN- $\gamma$ (Th1 response), and IL-4 (Th2 response). Steroid therapy has demonstrated good efficacy in stabilizing hemodynamics, shortening intensive care unit (ICU) stay and duration of mechanical ventilation, although without a clear beneficial effect on mortality, ${ }^{22}$ while the Infectious Diseases Society of America (IDSA) recommended against the use of corticosteroids in COVID19 based on data from previous coronaviruses in which no benefit was demonstrated. ${ }^{23}$

Both IL-1 and IL- 6 are responsible for elevation in acute-phase reactants, such as $\mathrm{C}$-reactive protein, serum amyloid $\mathrm{A}$, fibrinogen, and hepcidin, and inhibition of albumin synthesis which all together causes a pro-coagulant environment. In a meta-analysis including nine studies (total 1,426 patients), mean IL-6 levels were more than three times higher in patients with complicated COVID-19 compared to those with uncomplicated disease, and high IL- 6 levels were associated with mortality risk. ${ }^{24} \mathrm{IL}-6$ is an important marker of inflammation and can guide the clinicians in recognizing patients with severe COVID-19 early in the disease course.

However uncontrolled production of pro-inflammatory factors like IL-6, IL-8, IL-12, IL-1 $\beta$, IFN- $\gamma$, TGF $\beta$, and GM-CSF and chemokines like CCL2, CCL3, CCL5, CXCL9, CXCL10, and IL-10 together with reactive oxygen species leads to lung epithelial and endothelial cell apoptosis which damages lung microvascular and alveolar epithelial cell barrier, leading to vascular leakage, alveolar oedema, and hypoxia despite decreasing viral load after first week of illness. ${ }^{4,24}$

\section{ConCLUSiOn}

The aberrant release of multiple cytokines in COVID-19 produces immunopathogenic damage to tissues and organs, even while the immune response tries to overcome the evading mechanisms 
of virus. Delay in the interferon release and presence of LPS by secondary bacterial infections increases the severity of cytokine storm. IL- 6 could be used as a potential marker for severity of the ARDS. However, anti-IL6 antibody Tocilizumab failed to prove effective in clinical trials. Corticosteroid therapy is being given for moderate and severe cases of ARDS, but it needs a very fine balance to outweigh immunosuppressive effects.

\section{References}

1. Ye $Q$, Wang $B, M a o J$. The pathogenesis and treatment of the cytokine storm in COVID-19. J Infect 2020;80(6):607-613. DOI: 10.1016/j. jinf.2020.03.037.

2. Li H, Wang Y, Ji M, et al. Transmission route analysis of SARS-CoV-2: a systematic review and case report. Front Cell Dev Biol 2020;8:618. DOI: 10.3389/fcell.2020.00618.

3. Farcas GA, Poutanen SM, Mazzulli T, et al. Fatal severe acute respiratory syndrome is associated with multiorgan involvement by a coronavirus. J Infect Dis 2005;191(2):193-197. DOI: 10.1086/426870.

4. Peiris J, Chu C, Cheng V, et al. Clinical progression and viral load in a community outbreak of coronavirus associated SARS pneumonia: a prospective study. Lancet 2003;361(9371):767-772. DOI: 10.1016/ S0140-6736(03)13412-5.

5. Huang C, Wang Y, Li X, et al. Clinical features of patients infected with 2019 novel coronavirus in Wuhan, China. Lancet 2020;395(10223):497506. DOI: 10.1016/S0140-6736(20)30183-5.

6. Huang KJ, Su IJ, Theron M, et al. An interferon gamma related cytokine storm in SARS patients. J Med Virol 2005;75(2):185-194. DOI: 10.1002/ jmv.20255.

7. Teijaro JR, Walsh KB, Rice S, et al. Mapping the innate signaling cascade essential for cytokine storm during influenza virus infection. Proc Natl Acad Sci USA 2014;111(10):3799-3804. DOI: 10.1073/ pnas.1400593111.

8. Levy DE, Marie IJ, Durbin JE. Induction and function of type I and III interferon in response to viral infection. Curr Opin Virol 2011;1(6):476486. DOI: $10.1016 /$ j.coviro.2011.11.001.

9. Uze G, Monneron D. IL-28 and IL-29: newcomers to the interferon family. Biochimie 2007;89(6-7):729-734. DOI: 10.1016/j.biochi.2007.01.008.

10. Jensen S, Thomsen AR. Sensing of RNA viruses: a review of innate immune receptors involved in recognizing RNA virus invasion. J Virol 2012;86(6):2900-2910. DOI: 10.1128/JVI.05738-11.

11. Pahl HL. Activators and target genes of Rel/NF-kappaB transcription factors. Oncogene 1999;18(49):6853-6866. DOI: 10.1038/ sj.onc.1203239.

12. Overby AK, Popov VL, Niedrig M, et al. Tick borne encephalitis virus delays interferon induction and hides its double stranded RNA in intracellular membrane vesicles. J Virol 2010;84(17):8470-8483. DOI: 10.1128/JVI.00176-10.
13. He R, Leeson A, Andonov A, et al. Activation of AP-1 signal transduction pathway by SARS coronavirus nucleocapsid protein. Biochem Biophys Res Commun 2003;311(4):870-876. DOI: 10.1016/j. bbrc.2003.10.075.

14. Kopecky-Bromberg SA, Martinez-Sobrido L, Frieman M, et al. SARS coronavirus proteins Orf $3 \mathrm{~b}$, Orf 6 , and nucleocapsid function as interferon antagonists. J Virol 2006;81(2):548-557. DOI: 10.1128/ JVI.01782-06.

15. Ye $\mathrm{Y}$, Hauns $\mathrm{K}$, Langland JO, et al. Mouse hepatitis coronavirus A59 nucleocapsid protein is a type I interferon antagonist. J Virol 2007;81(6):2554-2563. DOI: 10.1128/JVI.01634-06.

16. Wathelet MG, Orr M, Frieman MB, et al. Severe acute respiratory syndrome coronavirus evades antiviral signaling: role of $n s p 1$ and rational design of an attenuated strain. J Virol 2007;81(21):1162011633. DOI: 10.1128/JVI.00702-07.

17. Züst R, Cervantes-Barragán L, Kuri T, et al. Coronavirus non-structural protein 1 is a major pathogenicity factor: implications for the rational design of coronavirus vaccines. PLoS Pathog 2007;3(8):e109. DOI: 10.1371/journal.ppat.0030109.

18. Kamitani W, Narayanan K, Huang C, et al. Severe acute respiratory syndrome coronavirus nsp1 protein suppresses host gene expression by promoting host mRNA degradation. Proc Natl Acad Sci USA 2006;103(34):12885-12890. DOI: 10.1073/pnas.0603144103. Describes a novel mechanism of viral protein-induced inhibition of host gene expression.

19. Tan YJ, Fielding BC, Goh PY, et al. Overexpression of $7 a$, a protein specifically encoded by the severe acute respiratory syndrome coronavirus, induces apoptosis via a caspase-dependent pathway. J Virol 2004;78(24):14043-14047. DOI: 10.1128/JVI.78.24.1404314047.2004.

20. Channappanavar R, Fehr AR, Vijay R, et al. Dysregulated type I interferon and inflammatory monocyte macrophage responses cause lethal pneumonia in SARS-CoV-infected mice. Cell Host Microbe 2016;19(2):181-193. DOI: 10.1016/j.chom.2016.01.007.

21. Hoffmann-La Roche's announcement about COVACTA's failure to meet study end points see https://www.roche.com/investors/ updates/invupdate-2020-07-29.htm.

22. Venkatesh B, Finfer $S$, Cohen J, et al. ADRENAL trial Investigators and the Australian-New Zealand intensive care society clinical trials group, adjunctive glucocorticoid therapy in patients with septic shock. N Engl J Med 2018;378(9):797-808. DOI: 10.1056/ NEJMoa1705835.

23. Bhimraj A, Morgan L, Shumaker RL, et al., 2020. Infectious Diseases Society of America Guidelines on the Treatment and Management of Patients With COVID-19. (Accessed June 28, 2020).

24. Grifoni E, Valoriani A, Cei F, et al. Interleukin- 6 as prognosticator in patients with COVID-19. J Infect 2020;81(3):452-482. DOI: 10.1016/j. jinf.2020.06.008. 